\title{
Evolutionary rate patterns of the Gibberellin pathway genes Yan-hua Yang ${ }^{1,2}$, Fu-min Zhang ${ }^{1}$ and Song Ge*1,2
}

Address: ${ }^{1}$ State Key Laboratory of Systematic and Evolutionary Botany, Institute of Botany, Chinese Academy of Sciences, Beijing 100093, PR China and ${ }^{2}$ Graduate University, Chinese Academy of Sciences, Beijing 100039, PR China

Email: Yan-hua Yang - yanhuayang@126.com; Fu-min Zhang - zhangfm@ibcas.ac.cn; Song Ge* - gesong@ibcas.ac.cn

* Corresponding author

Published: 18 August 2009

BMC Evolutionary Biology 2009, 9:206 doi:10.1 186//47|-2148-9-206

This article is available from: http://www.biomedcentral.com/l47I-2/48/9/206

(C) 2009 Yang et al; licensee BioMed Central Ltd.

This is an Open Access article distributed under the terms of the Creative Commons Attribution License (http://creativecommons.org/licenses/by/2.0), which permits unrestricted use, distribution, and reproduction in any medium, provided the original work is properly cited.
Received: 16 December 2008

Accepted: 18 August 2009

\begin{abstract}
Background: Analysis of molecular evolutionary patterns of different genes within metabolic pathways allows us to determine whether these genes are subject to equivalent evolutionary forces and how natural selection shapes the evolution of proteins in an interacting system. Although previous studies found that upstream genes in the pathway evolved more slowly than downstream genes, the correlation between evolutionary rate and position of the genes in metabolic pathways as well as its implications in molecular evolution are still less understood.
\end{abstract}

Results: We sequenced and characterized 7 core structural genes of the gibberellin biosynthetic pathway from 8 representative species of the rice tribe (Oryzeae) to address alternative hypotheses regarding evolutionary rates and patterns of metabolic pathway genes. We have detected significant rate heterogeneity among 7 GA pathway genes for both synonymous and nonsynonymous sites. Such rate variation is mostly likely attributed to differences of selection intensity rather than differential mutation pressures on the genes. Unlike previous argument that downstream genes in metabolic pathways would evolve more slowly than upstream genes, the downstream genes in the GA pathway did not exhibited the elevated substitution rate and instead, the genes that encode either the enzyme at the branch point (GA20ox) or enzymes catalyzing multiple steps (KO, KAO and GA3ox) in the pathway had the lowest evolutionary rates due to strong purifying selection. Our branch and codon models failed to detect signature of positive selection for any lineage and codon of the GA pathway genes.

Conclusion: This study suggests that significant heterogeneity of evolutionary rate of the GA pathway genes is mainly ascribed to differential constraint relaxation rather than the positive selection and supports the pathway flux theory that predicts that natural selection primarily targets enzymes that have the greatest control on fluxes.

\section{Background}

A primary goal of molecular evolutionary studies is to elucidate the driving forces governing evolutionary change and mechanisms of molecular evolution. A general pattern arising from previous studies is that genes or proteins varied substantially in their evolutionary rates, spanning more than 3 orders of magnitude [1-4]. Although many determinants, including functional, biophysical, and fitness-related variables $[3,5,6]$, have been proposed to explain such variation, what determines the evolutionary rate of a protein has been debated for decades and remains largely elusive despite some large-scale investiga- 
tions $[4,7,8]$. One possible reason for this is that many studies have focused on protein interaction networks that are very heterogeneous with different types of interactions $[5,8]$. False correlations in protein interaction networks and noise in biological data further complicate the analyses of protein evolution $[4,6]$. To study evolutionary rates and patterns of proteins in well-characterized metabolic networks or pathways has thus become an alternative for understanding of molecular evolution [9-15].

Analysis of molecular evolutionary patterns of different genes within metabolic pathways allows us to determine whether these genes are subject to equivalent evolutionary forces and how natural selection shapes the evolution of proteins in an interacting system. In studies of evolutionary rates of genes in the plant anthocyanin pathway, Rausher et al. [11] and Lu and Rausher [13] demonstrated that upstream genes in the pathway evolved more slowly than downstream genes both over a broad taxonomic distance involving monocots and dicots and at the intragenic level between species within a genus. They suggested that such difference in evolutionary rates between upstream and downstream genes was due to more constraint upon the upstream genes because they participated in several different biochemical pathways. The hypothesis that earlier acting genes in genetic pathways are under strong purifying selection has been confirmed by some studies (e.g., $[9,16])$ but not been supported by the others (e.g., [12] and see [17]).

Another hypothesis regarding protein evolution involves a theory of pathway fluxes, indicates that natural selection would target enzymes that control metabolic fluxes and thus where selection operates in a pathway will depend on the distribution of flux control among pathway genes $[17,18]$. It has been suggested that pathway branch points are usually the targets of selection because they might control the expression of biochemical phenotypes disproportionately $[10,14,18]$. A third alternative hypothesis argued that translational selection and selection for protein folding and design might govern the rate of protein sequence evolution [7,19-21]. Despite these arguments and some other attempts $[4,5,8,22]$, empirical studies with specific metabolic networks or pathways remain limited, particularly for plants.

Gibberellin (GA) biosynthetic pathway is involved in the production of gibberellins that control many aspects of plant growth and development, including seed germination, stem elongation, leaf expansion, and flower and seed development $[23,24]$. Despite a hundred GAs identified from plants, a small number of them such as $\mathrm{GA}_{1}$ and $\mathrm{GA}_{4}$ are found to function as bioactive hormones. These bioactive GAs are synthesized from trans-geranylgeranyl diphosphate (GGDP) through the 12-step conversion
(Figure 1). To date 7 enzymes have been identified to be responsible for GA biosynthesis and function in multiple locations within the cell, involving the chloroplast, the ER membrane, and the cytoplasm. These 7 enzymes can be divided into 2 groups, i.e., the enzymes catalyzing early steps: ent-copalyl diphosphate synthase (CPS), ent-kaurene synthase (KS), ent-kaurene oxidase (KO), and entkaurenoic acid oxidase (KAO) and those for later steps: GA20-oxidase (GA20ox), GA3-oxidase (GA3ox), and GA2-oxidase (GA2ox) (Figure 1) [23]. In addition to a well-defined pathway, almost all of the genes encoding the 7 metabolic enzymes and their related mutants have been isolated and identified $[23,24]$. Therefore, the GA biosynthetic pathway provides an excellent system for exploring the correlation between evolutionary rate and position of the genes in metabolic pathways as well as its implications in molecular evolution. In addition, the GA biosynthetic pathway is also a good system to investigate the relative importance of selective constraint and positive selection as well as how adaptive variation is distributed among proteins in metabolic pathways. In this study, we isolated and sequenced portions of the 7 GA pathway genes from representative species of the rice tribe and analyzed their rates and patterns of molecular evolution. Our specific goals were 1) to compare evolutionary rates across the GA pathway genes and ask if evolutionary rate of the genes depends on its function or gene's position within the pathway; 2) to examine whether the GA pathway genes exhibit evidence of positive selection and particularly to understand the relative contribution of selective constraint and positive selection to variation of evolutionary rates of the GA pathway genes.

\section{Methods \\ Plant materials and genes analyzed}

We isolated and sequenced portions of all 7 GA pathway genes from members of the rice tribe (Oryzeae). Phylogeny of the rice tribe has been well resolved by previous studies based on sequences of plastid, mitochondrial and nuclear genes $[25,26]$ and provided an important framework for molecular evolution study for this group (Additional file 1). To analyze the patterns of molecular evolution of the GA pathway genes, 8 diploid species were selected to represent major phylogenetic lineages of Oryzeae that are separated by a range of genetic distances [26]. They consisted of 5 Oryza species representing 5 diploid genome types, Oryza sativa (A), O. officinalis (C), O. australiensis $(\mathrm{E}), O$. brachyantha $(\mathrm{F}), \mathrm{O}$. granulata $(\mathrm{G})$, and one each of other 3 genera in the tribe (Chikusichloa aquatica, Luziola leiocarpa, and Rhynchoryza subulata). One species in the tribe Ehrhartoideae that is sister to Oryzeae, Ehrharta erecta, was used as an outgroup $[26,27]$.

Of 7 enzymes in the GA biosynthetic pathway, 4 enzymes in the early steps (CPS, KS, KO and KAO) were encoded 


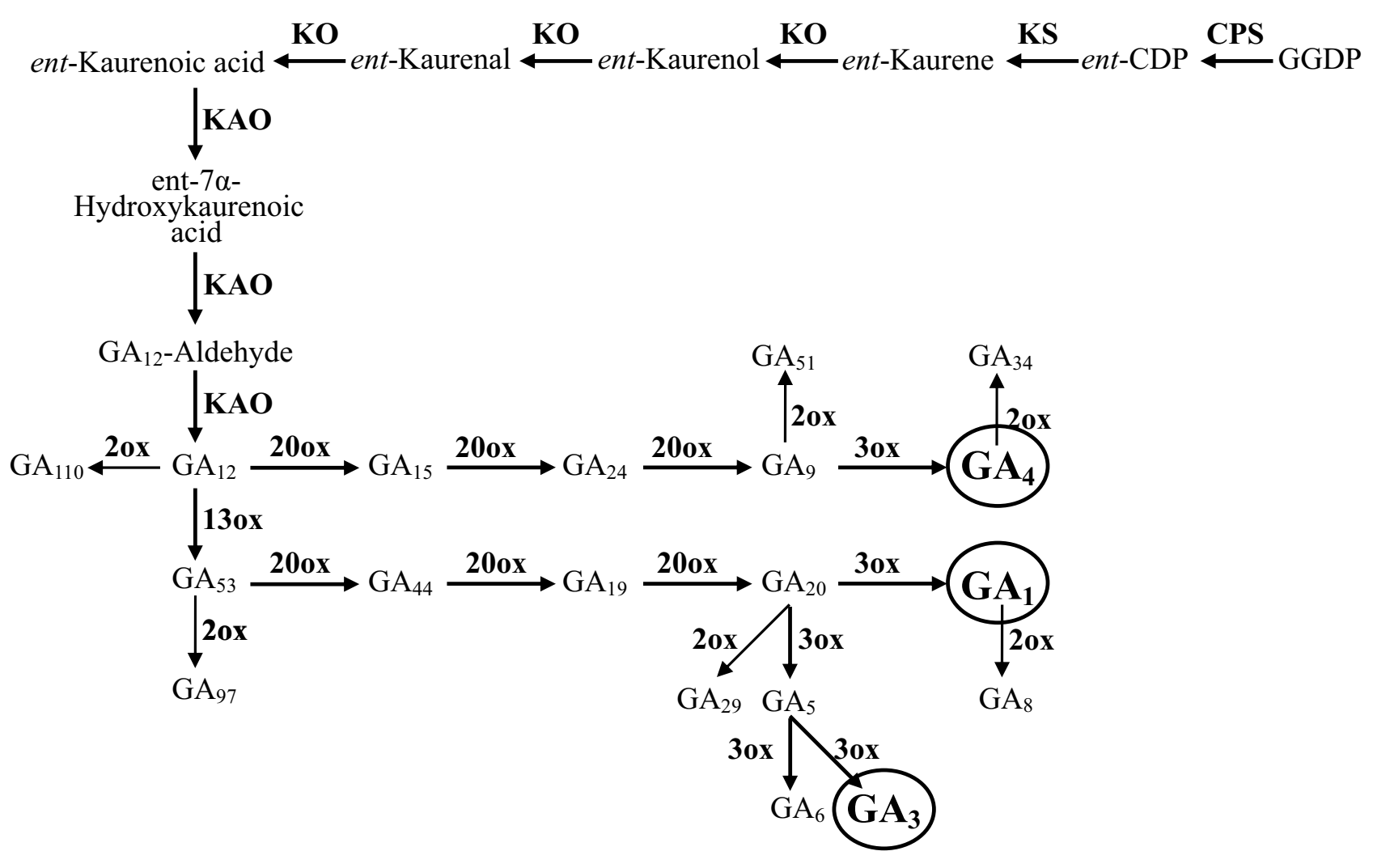

Figure I

Simplified GA biosynthetic pathway in plants. The shaded metabolites have been demonstrated to function as bioactive hormones.

by single-copy genes (CPS1, KS1, KO2 and KAO) [23] and sampled in this study. Because the 3 enzymes in the later steps (GA20ox, GA3ox and GA2ox) were encoded by small gene families $[23,24]$, we chose to sequence GA200x2, GA30x2 and GA20x4 that were highly expressed in all organs of the wild-type rice [23]. Previous studies showed that mutants with all 7 enzymes except for GA2ox exhibited a typical phenotype of dwarfism, indicating the functional importance of these enzymes in GA biosynthesis [23]. Detailed information of the genes and the sequences and positions of the primers are listed in table 1 and Additional file 2, respectively. Species sampled and the GenBank accession numbers of the sequences obtained in this study are listed in Additional files 3 and 4 , respectively.

\section{DNA extraction, amplification and sequencing}

Total DNA was isolated from silica-gel dried or fresh leaves using the method as described by Ge et al. [25]. Because the DNA fragments of each gene contained multiple exons and introns, additional internal primers were used for sequencing of four genes (CPS1, KS1, KO2 and $K A O)$ that were about $2 \mathrm{~kb}$ in length. Their sequences and melting temperatures ( $\mathrm{Tm}$ ) are provided in Additional file 5. Polymerase chain reaction (PCR) was performed in a total volume of $25 \mu \mathrm{l}$ which contained 5-50 ng of template DNA, $0.2 \mu \mathrm{M}$ of each primer, $200 \mu \mathrm{M}$ of each dNTP, $10 \mathrm{mM}$ Tris-Cl (pH 8.3), $50 \mathrm{mM} \mathrm{KCl}, 1.5 \mathrm{mM} \mathrm{MgCl}_{2}$, and 0.75 U ExTaq DNA polymerase (TaKaRa, Shiga, Japan). Amplification was carried out in a T gradient $96 \mathrm{U}$ thermocycler (Biometre, Göttingen, Germany) as follows: 3 min at $94^{\circ} \mathrm{C}$, followed by 33 cycles of $30 \sim 35 \mathrm{~s}$ at $94^{\circ} \mathrm{C}$, $30 \sim 35 \mathrm{~s}$ at $54^{\circ} \mathrm{C} \sim 58^{\circ} \mathrm{C}, 2 \mathrm{~min} 30 \mathrm{~s}$ at $72^{\circ} \mathrm{C}$ and a final extension at $72^{\circ} \mathrm{C}$ for $10 \mathrm{~min}$ (CPS1, KS1, KO2, and $K A O)$. Because GC contents of GA20ox2, GA3ox2 and GA2ox4 were as high as about 70\%, routine PCR amplification did not work well. We performed the amplification using LA Taq DNA polymerase, special GC Buffer I and dNTP Mixture (TaKaRa, Shiga, Japan). Except for annealing temperature at $60^{\circ} \mathrm{C}$, the protocol was basically similar to the above protocol. All PCR products were separated by electrophoresis on $1.5 \%$ agarose gels stained with ethidium bromide. After purification using DNA Purification kit, most of amplification products were sequenced directly. For those PCR products that were weakly amplified and difficult to be sequenced directly, we used the 
Table I: Information on the genes and sequences of the primers used in the study.

\begin{tabular}{|c|c|c|c|c|c|c|}
\hline Gene & Location $^{a}$ & Enzyme & $\begin{array}{l}\text { Aligned coding I } \\
\text { ength (bp) }\end{array}$ & GC $(\mathrm{GC} 3)^{b}$ & $\mathrm{ENC}^{b}$ & Primer sequences $^{c}$ \\
\hline CPSI & 2 & ent-copalyl diphosphate synthases I & 1002 & $0.462(0.494)$ & 54.6 & $\begin{array}{l}\text { FI: AACTTGTGGAGGTTAGCAG } \\
\text { F2: TGTGGAGGYTAGCRGAGG } \\
\text { RI: AAGTCGCTCAGAGGCACG } \\
\text { R2: TAGCCCATGCAAGTCGCTC }\end{array}$ \\
\hline KSI & 4 & ent-kaurene synthase I & 1023 & $0.426(0.417)$ & 52.8 & $\begin{array}{l}\text { FI: TGCTGAAGCTTCCAGTTTCC } \\
\text { F2: TCCAGTTTCCGTGAATCAC } \\
\text { RI: CTTGCACATCTTCCAGAAC } \\
\text { R2: CCTTGACGACTGCATTCAC }\end{array}$ \\
\hline KO2 & 6 & ent-kaurene oxidase II & 1050 & $0.514(0.639)$ & 58.2 & $\begin{array}{l}\text { F: CTGTAGTTGTGCTCAATTC } \\
\text { RI: GCCATCGTCTTGTACATGTC } \\
\text { R2: TCAGCCTCCACYCGAACTC }\end{array}$ \\
\hline KAO & 6 & ent-kaurenoic acid oxidase & 1053 & $0.603(0.820)$ & 39.2 & $\begin{array}{l}\text { F: CAGGACGTTCATGTTCAGCAG } \\
\text { RI: TCGTCGCCAAGCAGTTGTC } \\
\text { R2: GCCAAGCAGTTGTCCAC }\end{array}$ \\
\hline GA20ox2 & I & GA20-oxidase II & 597 & $0.714(0.966)$ & 30.1 & $\begin{array}{l}\text { F: ATCCCGGAGCCATTCGTSTG } \\
\text { R: TGAAGGTGTCGCCGATGTTG }\end{array}$ \\
\hline GA3ox2 & I & GA3-oxidase II & 786 & $0.710(0.965)$ & 30.4 & $\begin{array}{l}\text { FI: ACCCGCTCTRCTTCGACTTC } \\
\text { F2: } \\
\text { GGCGGGTGCCGGAGACGCACG } \\
\text { R: CCATGTACTCSGGCCACGTGAC }\end{array}$ \\
\hline GA2ox4 & 5 & GA2-oxidase IV & 819 & $0.694(0.926)$ & 34.0 & $\begin{array}{l}\text { F: GAGCAGATCTCGCTGSTGAG } \\
\text { RI: CAGGCGGTTGTCGCYGAG } \\
\text { R2: AGGCGGGAGAGGTAGGCAG }\end{array}$ \\
\hline Total & & & 6330 & & & \\
\hline
\end{tabular}

${ }^{a}$ Chromosomal locations in 0 . sativa.

bAverage of 8 Oryzeae species.

cLocations of the primers for each fragment are showed in Additional file 2. The internal primers were used for cycle sequencing are listed in Additional file 5 .

pGEM-T Vector (Promega Corporation, Madison, USA) to clone and sequence at least three clones. Sequencing was carried out on a Megabase 1000 automatic DNA sequencer (Amersham Pharmacia Biotech). All sequences have been deposited in GenBank, and their accession numbers are EF577637 EF577669, EU179376 EU179435 (Additional file 4).

\section{Sequence analyses}

Sequences were translated into the predicted amino acid sequence and aligned using ClustalX with version 1.81 [28], followed by manual adjustment. Excluding intron regions, the stretches of amino acid residues were conserved well enough across eight species to perform unambiguous alignments. The possibility of sequence saturation was examined using DAMBE 4.5.45 [29]. A plot of the number of transitions and transversions vs. divergence offers a visual display of substitution saturation, with an asymptotic relationship indicating the presence of saturation [29]. Pairwise number of synonymous and nonsynonymous substitutions per site $\left(d_{\mathrm{S}}\right.$ and $\left.d_{\mathrm{N}}\right)$ as well as nonsynonymous/synonymous substitution rate ratio $\left(d_{\mathrm{N}} / d_{\mathrm{S}}\right)$ between the eight species were calculated for the coding regions of all 7 genes using the improved approximate method of Yang and Nielsen [30], implemented in YNO0 (CODEML) of PAML version 4 [31]. This method considers two important evolutionary features of DNA sequence: transition/transversion rate bias and codon frequency bias. Based on the pruned phylogenetic tree of the eight species (Additional file 6), we also estimated the $d_{\mathrm{N}} /$ $d_{\mathrm{S}}$ value of each gene using CODEML of the PAML version $4[31]$.

The magnitude of codon bias is often used to reflect the degree of selective constraint in a gene and variation of synonymous substitution rates among genes may be related to codon usage $[32,33]$. To measure the extent of codon usage bias, we estimated effective number of codons (ENC) using DnaSP version 4.10.9 [34], which varies between 20 and 61, with the lower the value, the more biased codon usage [35].

\section{Comparing $\mathrm{d}_{S}$ and $\mathrm{d}_{N}$ among genes}

The pairwise synonymous and nonsynonymous genetic distances of 8 species were calculated for each gene using the method of Yang and Nielsen [30]. Of total 28 species pairs, we chose 15 species pairs involving 5 Oryza species and other three representatives of the rice tribe to represent maximum divergence time (20 myr, Additional file $1)$. To evaluate the reliability of estimated genetic distances of the 15 pairs, we checked the distance variance of each pair of the genes and found that the pairs involving 
O. sativa or O. brachyantha have high variance for GA20ox, GA3ox and GA2ox. Thus six species pairs involving either of the two species were excluded. Based on the rest 9 species pairs (O. officinalis vs. C. aquatica, O. officinalis vs. $L$. leiocarpa, O. officinalis vs. R. subulata, O. australiensis vs. C. aquatica, O. australiensis vs. L. leiocarpa, O. australiensis vs. R. subulata, O. granulata vs. C. aquatica, O. granulata vs. L. leiocarpa, and O. granulata vs. R. subulata), the mean distance and its standard error of each gene were calculated to show variation of $d_{\mathrm{S}^{\prime}} d_{\mathrm{N}}$ among genes. To compare $d_{\mathrm{S}}$ and $d_{\mathrm{N}}$ among genes, we calculated the $d_{\mathrm{S}}$ and $d_{\mathrm{N}}$ for each branch of the pruned tree of the six species (Additional file 1 and Additional file 6) using CODEML of the PAML version 4 [31]. Of the tree, there are total 10 evolutionary independent branches (Additional file 6). Two data matrices for seven genes were obtained for $d_{\mathrm{S}}$ and $d_{\mathrm{N}}$, respectively. We used two approaches to detect whether evolutionary rates differed among the genes. First, we used multiple range test and Fisher's least significant difference (LSD) procedure to discriminate the means of the genetic distances of 9 species pairs among 7 genes. To compare $d_{\mathrm{S}}$ and $d_{\mathrm{N}}$ among genes, we calculated the $d_{\mathrm{S}}$ and $d_{\mathrm{N}}$ for each branch of the pruned tree including six species (Additional file 6) using CODEML of the PAML version 4 [31]. On the tree, there are 10 evolutionarily independent branches (Additional file 6). Then we used Wilcoxon signed rank test [36] in the pairwise comparisons of distances of the 10 branches between genes to test significant difference among the GA genes.

\section{Comparing $\mathrm{d}_{N} / \mathrm{d}_{S}$ among genes}

Pairwise $d_{\mathrm{N}} / d_{\mathrm{S}}$ ratios of 8 species were estimated for each gene using the method of Yang and Nielsen [30]. The mean $d_{\mathrm{N}} / d_{\mathrm{S}}$ ratios of 9 species pairs and standard errors were calculated to show variation of $d_{\mathrm{N}} / d_{\mathrm{S}}$ among genes. We used a method described by Lu and Rausher [13] to compare $d_{N} / d_{S}$ ratios for the seven genes. For this analysis, we first estimated a single value of $d_{\mathrm{N}} / d_{\mathrm{S}}$ for each gene using the model M0 of by CODEML of PAML version 4 [31]. We then detected the significance of differences of $d_{\mathrm{N}} / d_{\mathrm{S}}$ values between genes by comparing the likelihood of the model using the estimated value of $d_{\mathrm{N}} / d_{\mathrm{S}}$ to the likelihoods of the same model and using $d_{\mathrm{N}} / d_{\mathrm{S}}$ constrained to various values [13].

\section{Detection of positive selection}

The ratio of nonsynonymous to synonymous substitution rate $\left(\omega=d_{\mathrm{N}} / d_{\mathrm{S}}\right)$ provides an effective measure to detect selection or selective pressure on a gene or gene region, with $\omega<1,=1$, and $>1$ indicating purifying selection, neutral evolution, and positive selection, respectively $[31,37]$. We performed likelihood-based analyses using the CODEML program of PAML version 4 [31] to explore the selective processes acting on the GA biosynthetic genes. First, we tested whether the evolutionary rates of each gene in the pathway differed among lineages within the gene tree by using the branch models. The one ratio model (M0) assumes a single $\omega$ for all branches and all sites, whereas the free ratio model (Mf) assumes an independent $\omega$ ratio for each branch of the tree. A likelihood ratio test (LRT) was conducted to determine whether there was statistically significant difference between two models. If the LRT is significant, the null hypothesis that two models are not significantly different is rejected, and the model with higher likelihood value is assumed to be a better model [38,39].

We next used site-specific models to examine whether particular amino acid residues were subject to positive selection because the branch test often has little power to detect positive selection due to the fact that the $\omega$ ratio is seldom detected greater than 1 if all the sites are averaged [40]. The nested codon models $[39,40]$ were performed for each gene separately. In addition to one ratio model (M0), the neutral model (M1a) classifies all the sites into 2 categories, one under strict constraint $(0<\omega<1)$ (purifying selection) and the other under neutral $(\omega=1)$. Positive selection model (M2a) is based on M1a and assumes a third category under positive selection $(\omega>1)$. Beta model (M7) assumes a beta distribution of the $\omega$ ratios, and beta\& $\omega$ model (M8) extends an independent ratio estimated by the data. Models assuming positive selection M8 and M2a are compared with null models M7 and M1a, respectively. Positive selection is invoked if the LRT is significant and there is site with $\omega>1[39,40]$.

\section{Results}

Sequences of the 7 GA pathway genes were isolated from all sampled Oryzeae species and an outgroup, Ehrharta erecta. The sequenced regions of the 7 genes ranged from 699 bp to 2231 bp in rice and their aligned coding regions varied between 597 bp and 1053 bp for species used in this study (table 1 and Additional file 4). The GC contents for the total and 3 individual codon positions were similar for the same gene across species but varied greatly among genes, with the means ranging from 0.426 (KS1) to 0.714 (GA20ox2) (table 1 and Additional file 4). It is noted that GC contents of the 3 genes at the later steps were much higher than those of the 4 genes at the early steps in the pathway, particularly at the 3rd position (GC3) where the averages were $0.926 \sim 0.966$ for the genes at the later steps in contrast to the values of $0.417 \sim$ 0.82 for the genes at the early steps (table 1 and Additional file 4). To estimate accurately and compare substitution rate and $\omega$ ratios of different GA pathway genes, we evaluated sequence saturation of substitution for each of the seven genes. Results of saturation plots did not reveal any mutational saturation for all the seven GA genes, indicating that the datasets were suitable for further analyses of the differences of substitution rate and $\omega$ ratios. 


\section{Comparing $\mathrm{d}_{\mathbf{N}}$ and $\mathrm{d}_{\mathrm{S}}$ among genes}

The averages of $d_{\mathrm{S}}$ and $d_{\mathrm{N}}$ of the 9 species pairs for each gene were shown in Figure 2 and table 2. Both $d_{\mathrm{S}}$ and $d_{\mathrm{N}}$ values varied greatly among genes, with the highest $d_{\mathrm{S}}$ (0.747 for GA2ox4) being 3.5 times the lowest $(0.214$ for KS1) and the highest $d_{\mathrm{N}}(0.086$ for KS1) being 5.9 times the lowest ( 0.015 for GA20ox2). For $d_{S}$, the 3 genes in the early steps of the pathway (CPS1, KS1 and KO2) have significantly lower $d_{\mathrm{S}}$ values than $K A O$ and 3 genes at the later steps of the pathway (GA20ox2, GA3ox2 and GA2ox4). For $d_{\mathrm{N}^{\prime}}$ GA20ox2 has the lowest value (0.015), followed by 4 genes (KO2, GA3ox2, CPS1, and KAO), and the second and last genes in the pathway, KS1 and GA2ox4, have the highest and comparable $d_{\mathrm{N}}$ value (0.086 and 0.087 ). Unlike $d_{\mathrm{S}}$ values, $d_{\mathrm{N}}$ varied significantly both between and within genes in the early and late steps of the pathway. It is noted that 2 types of substitutions did not correlated $\left(\mathrm{r}^{2}=0.009, P=0.840\right)$, which is clearly reflected

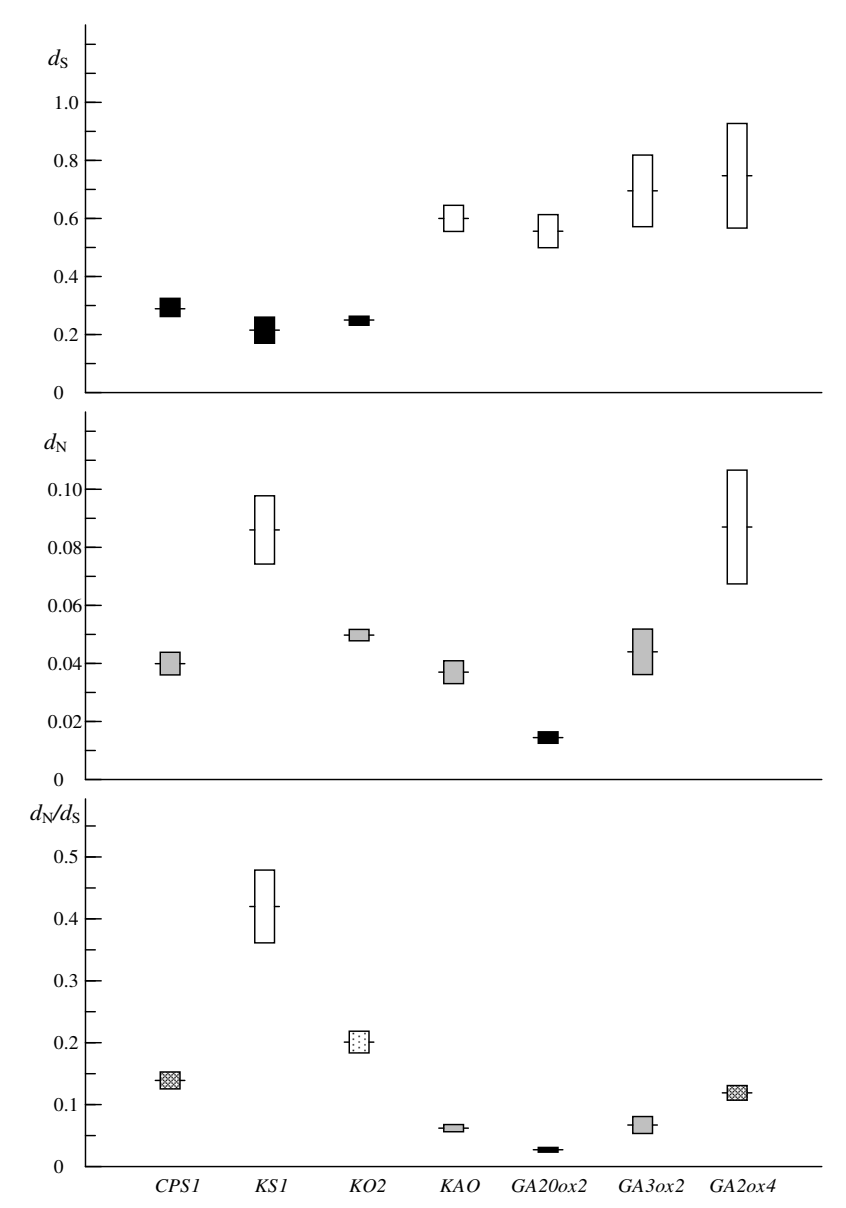

Figure 2

Evolutionary rates at sysnonymous and nonsynonymous sites of the GA pathway genes. Horizontal bars indicate the averages of $d_{S}, d_{N}$ and $d_{S} / d_{N}$ values. Boxes represent $95 \%$ confidence intervals. by the fact that KS1 had a highest $d_{\mathrm{N}}$ value but the lowest $d_{\mathrm{S}}$ (Figure 2 and table 2). Multiple range tests generated 2 and 3 homogenous groups of genes for $d_{\mathrm{S}}$ and $d_{\mathrm{N}}$, respectively, with statistically significant difference between genes among groups and non-significant difference between genes within groups (table 2 ). To overcome the rate heterogeneity of $d_{\mathrm{S}}$ and $d_{\mathrm{N}}$ among lineages, we performed the Wilcoxon signed rank test for pairwise comparisons of $d_{\mathrm{S}}$ and $d_{\mathrm{N}}$ between genes for 10 independent branch of pruned tree consisting of 6 species. For $d_{S^{\prime}}$ the gene KAO and three late-step genes (GA20ox2, GA3ox2, GA2ox4) showed significant rate differences from the first 3 genes in the pathway (CPS1, KS1 and KO2) $(P<0.01)$. No significant difference was detected between $K A O$ and the late-step genes and among the early-step genes. For $d_{\mathrm{N}^{\prime}}$ none of the tests showed significant difference, involving the comparisons between and within both early- and latestep genes (table 2 and Additional file 7).

\section{Comparing $\mathrm{d}_{N} / \mathrm{d}_{S}$ among genes}

The $d_{\mathrm{N}} / d_{\mathrm{S}}$ ratios of the 9 species pairs varied remarkably among the 7 genes, with the highest value ( 0.420 for KS1) being 15.6 times the lowest (0.027 for GA20ox2) (Figure 2 and table 2 ). We compared $d_{\mathrm{N}} / d_{\mathrm{S}}$ ratios for the 7 genes and detected the significance of differences between genes using the method of Lu and Rausher [13]. The $d_{\mathrm{N}} / d_{\mathrm{S}}$ value for each gene ranged from 0.386 (KS1) to 0.022 (GA20ox2) with a trend similar to that estimated by pairwise $d_{\mathrm{N}} / d_{\mathrm{S}}$ ratios (table 2 ). Based on log-likelihood ratio comparisons, we found 4 homogenous groups, i.e., A (GA20ox2), B (KAO and GA3ox2), C (KO2, CPS1, and GA2ox4), and D (KS1). As shown in table 3, significant difference of $d_{\mathrm{N}} / d_{\mathrm{S}}$ ratios is found between genes from different groups but not found between genes from the same groups. The results are similar to those obtained by multiple range test and Wilcoxon signed rank test, with the exception that KO2 and CPS1 were in the same group in log-likelihood ratio comparisons (table 3).

\section{Codon usage bias and its correlation with GC3 and substitution rates}

Estimates of the codon usage indicated that the ENC for the same gene was similar across all species ( $\chi^{2}$ test, $P=$ 1.00) (Additional file 4), suggesting that the magnitude of codon bias underwent little change during the diversification of the rice tribe. However, substantial variation was found among genes in the degree of codon bias, with the means of ENC ranging from 30.1 (GA20ox2) to 58.2 (KO2) (table 1 and Additional file 4). Pairwise comparisons of one-way ANOVA found that differences of the ENC were significant for all pairwise comparisons $(P<$ $0.001)$ except for CPS1 vs. KS1 $(P=0.090)$ and GA20ox2 vs. GA3ox2 $(P=0.386)$. Note that the 3 genes in the late steps (GA20ox2, GA3ox2, and GA2ox4) exhibited strong codon usage bias $(\mathrm{ENC}=30.1 \sim 34.0)$, whereas the 3 
Table 2: Tests on difference of $d_{S}, d_{N}$ and $d_{N} / d_{S}$ among the $7 \mathrm{GA}$ genes ${ }^{a}$

\begin{tabular}{|c|c|c|c|c|c|c|}
\hline \multirow[t]{2}{*}{ Gene } & \multicolumn{2}{|c|}{$d_{S}$} & \multicolumn{2}{|c|}{$d_{N}$} & \multicolumn{2}{|c|}{$d_{N} / d_{S}$} \\
\hline & Mean \pm SEb & WSRTc & Mean \pm SEb & WSRTc & Mean \pm SE & $\omega^{d}$ \\
\hline CPSI & $0.292 \pm 0.016$ & $A$ & $0.040 \pm 0.002$ & B & $0.139 \pm 0.007$ & $C(0.144)$ \\
\hline KSI & $0.214 \pm 0.023$ & $A$ & $0.086 \pm 0.006$ & $\mathrm{C}$ & $0.420 \pm 0.030$ & $D(0.386)$ \\
\hline KO2 & $0.250 \pm 0.008$ & $A$ & $0.050 \pm 0.001$ & B & $0.201 \pm 0.009$ & $C(0.177)$ \\
\hline$K A O$ & $0.600 \pm 0.023$ & $B$ & $0.037 \pm 0.002$ & B & $0.062 \pm 0.003$ & $\mathrm{~B}(0.074)$ \\
\hline GA20ox2 & $0.556 \pm 0.029$ & B & $0.015 \pm 0.001$ & A & $0.027 \pm 0.002$ & $A(0.022)$ \\
\hline GA3ox2 & $0.699 \pm 0.063$ & B & $0.044 \pm 0.004$ & B & $0.066 \pm 0.007$ & $B(0.073)$ \\
\hline GA2ox4 & $0.747 \pm 0.092$ & B & $0.087 \pm 0.010$ & C & $0.119 \pm 0.006$ & $C(0.129)$ \\
\hline
\end{tabular}

a Capital letters (A to $D)$ indicate the homogenous groups.

b Calculated based on 9 specie pairs.

c Wilcoxon signed rank test using 10 independent branches of the pruned tree consisting of 6 species

${ }^{d} \omega$, calculated as $d_{N} / d_{S}$ ratios using PAML model M0.

genes in the early steps (CPS1, KS1 and KO2) possessed little codon usage bias ( $\mathrm{ENC}=52.8 \sim 58.2$ ). $K A O$ exhibits an intermediate degree of codon usage bias with ENC of 39.2. ANOVA showed that ENC of the late-step genes differed significantly from those of the early step genes $(P<$ 0.001 ). To further investigate the correlation between evolutionary rate and expression level, we analyzed the correlation between each of three parameters $\left(d_{\mathrm{N}}, d_{\mathrm{S}}\right.$, and $d_{\mathrm{N}} /$ $d_{\mathrm{S}}$ ) and ENC that negatively correlates with expression level. Results showed that significantly negative correlation existed between $d_{\mathrm{S}}$ and ENC $(P<0.001)$, but not between ENC and either $d_{\mathrm{N}}(P=0.540)$ or $d_{\mathrm{N}} / d_{\mathrm{S}}(P=$ 0.103).

\section{Testing for positive selection}

Significant rate heterogeneity among the GA pathway genes, particularly for $d_{\mathrm{N}} / d_{\mathrm{S}}$ ratios that span more than one order of magnitude (Figure 2), might result either from intense purifying selection on slowly evolving genes (e.g., GA20ox2) or from frequent episodes of positive selection on fast evolving genes (e.g., KS1). Therefore, we first used branch models to test whether the evolutionary rate of each gene differed among lineages within the rice tribe. For all 7 genes, free ratio model (Mf) did no have significantly higher likelihood scores than one ratio model $(\mathrm{M0})(P>0.05)$ (Additional file 8$)$. The $\omega$ values were estimated to be $0.022 \sim 0.386$ under M0 model, sug-

Table 3: Comparison and significant test of log-likelihood ratio of $d_{N} / d_{S}$ between genes

\begin{tabular}{|c|c|c|c|c|c|}
\hline Category/Gene & Estimated $d_{N} / d_{S}$ & $\ln L$ & Fixed $d_{N} / d_{S}$ & $\operatorname{lnL}$ & $2 \Delta \mathrm{L}^{\mathrm{a}}$ \\
\hline \multicolumn{6}{|l|}{ Between groups $D$ and $C$} \\
\hline KSI & 0.386 & -3341.16 & 0.2815 & -3345.46 & $8.59 * *$ \\
\hline KO2 & 0.177 & -3118.44 & 0.2815 & -3126.22 & $15.56 * * *$ \\
\hline CPSI & 0.144 & -3034.22 & 0.2815 & -3049.87 & $31.3 * * *$ \\
\hline GA2ox4 & 0.129 & -2733.05 & 0.2815 & -2755.45 & $44.8 * * *$ \\
\hline \multicolumn{6}{|l|}{ Within group C } \\
\hline KO2 & 0.177 & -3118.44 & 0.153 & -3119.19 & $1.50 \mathrm{NS}$ \\
\hline CPSI & 0.144 & -3034.22 & 0.153 & -3034.35 & $0.26 \mathrm{NS}$ \\
\hline GA2ox4 & 0.129 & -2733.05 & 0.153 & -2734.14 & $2.18^{\mathrm{NS}}$ \\
\hline \multicolumn{6}{|c|}{ Between groups $C$ and $B$} \\
\hline KO2 & 0.177 & -3118.44 & 0.1015 & -3128.93 & $20.98 * * *$ \\
\hline CPSI & 0.144 & -3034.22 & 0.1015 & -3038.05 & $7.66^{* *}$ \\
\hline GA2ox4 & 0.129 & -2733.05 & 0.1015 & -2735.16 & $4.21^{*}$ \\
\hline KAO & 0.074 & -3071.99 & 0.1015 & -3075.8 & $7.62^{* *}$ \\
\hline GA3ox2 & 0.073 & -2210.46 & 0.1015 & -2213.21 & $5.50^{*}$ \\
\hline \multicolumn{6}{|l|}{ Within group B } \\
\hline KAO & 0.074 & -3071.99 & 0.0735 & -3071.99 & ONS \\
\hline GA3ox2 & 0.073 & -2210.46 & 0.0735 & -2210.46 & ONS \\
\hline \multicolumn{6}{|c|}{ Between groups $B$ and $A$} \\
\hline KAO & 0.074 & -3071.99 & 0.0475 & -3078.8 & $13.62^{* * * *}$ \\
\hline GA3ox2 & 0.073 & -2210.46 & 0.0475 & -2214.72 & $8.52^{* *}$ \\
\hline GA20ox2 & 0.022 & -1373.16 & 0.0475 & -1379.06 & $11.8^{* * * *}$ \\
\hline
\end{tabular}

a d.f. $=1, * * * P<0.001, * * P<0.01, * P<0.05$, Ns not significant. 
gesting that purifying selection or selection constraint best explains the evolution of these genes.

Because the branch model test averages the ratio across all sites and is a very conservative test of positive selection $[39,40]$, we applied site-specific codon models to the 7 genes separately, to determine whether there was positive selection on codon sites of these genes. Results showed that, for all 7 genes, models M2a and M8 assuming positive selection were not significantly better than the null models M1a and M7 (for M1a vs. M2a, $2 \Delta \mathrm{L}=0.0 \sim 3.72$, $0.1<P \leq 1.0$; for $\mathrm{M} 7$ vs. $\mathrm{M} 8,2 \Delta \mathrm{L}=0.0 \sim 5.3,0.05<P \leq 1.0$ ) (Additional file 8). Consequently, all 7 genes are under strong selective constraint, which excludes the possibility of past episodes of positive selection on these genes.

\section{Discussion \\ Evolutionary rate and position of genes in the pathway}

We used two different approaches to have detected significant rate heterogeneity among 7 GA pathway genes for both synonymous and nonsynonymous sites. Multiple range tests classified the 7 genes into 2 and 3 homogenous groups of synonymous and nonsynonymous sites, respectively, with significant rate difference among genes between groups but not among genes within groups. It is noteworthy that the patterns of rate heterogeneity were different for 2 types of substitutions. For $d_{\mathrm{S}}$, the late-step genes (GA20ox2, GA3ox2, and GA2ox4) have significantly higher substitution rates than the early-step genes (CPS1, $K S 1, K O 2$, and $K A O)(P<0.05)$, whereas one early-step gene (KS1) and one late-step gene (GA2ox4) consist of one homogenous group with the highest $d_{\mathrm{N}}$.

It is well established that large variation in among-gene substitution rates might be determined by two factors: the rate of mutation and the intensity of selection $[1,5,41]$. To distinguish these two possibilities, we first asked whether there was regional similarity for $d_{\mathrm{S}}$ and $d_{\mathrm{N}}$ values because mutation pressure would lead to region-specific mutation rates $[1,42]$. It is evident that genes in the same homogenous $d_{\mathrm{S}}$ or $d_{\mathrm{N}}$ groups locate on different chromosomes (table 1). These observations, in conjunction with the lack of correlation between $d_{\mathrm{S}}$ and $d_{\mathrm{N}^{\prime}}$ do not support the mutation-driven hypothesis. To further investigate possible role of mutation, we calculated the GC content of noncoding sites of the 7 genes (Additional file 4). If difference of GC3 was caused by mutation pressure, we would expect a positive correlation between GC3 of coding sites and GC of noncoding sites because the latter is expected to reflect differences in mutation rate $[2,5]$. The result did not find a significant correlation between them $\left(\mathrm{r}^{2}=0.516, P=\right.$ 0.069), suggesting that mutation bias alone cannot explain the differences of codon usage in the 7 genes. Consequently, differences of selection intensity are most likely to account for the substitution rate variation in the
GA pathway genes, though differential mutation pressures on genes cannot be excluded entirely.

Rausher et al. [11] studied evolution patterns of 6 core structural genes in the anthocyanin pathway and found that, over a broad taxonomic distance involving monocots and dicots, the upstream genes evolved substantially more slowly than the downstream genes. They thus hypothesized that the upstream genes in a metabolic pathway evolved more slowly than the downstream genes due to stronger purifying selection for the upstream genes. Such a pattern has also been confirmed at the intragenic [13] and population levels [15] in the genus Ipomoea as well as by a molecular population study on regulatory and signal transduction genes [9] but was not supported by other studies $[8,12,17]$. In the GA metabolic pathway, we did not find the correlation between substitution rate and position of genes. Notably, 2 genes (KS1 and GA20x4) with the highest $d_{\mathrm{N}}$ belong to the early and later acting genes, respectively (Figure 1). In addition, 3 late-step genes have significantly lower $d_{\mathrm{N}} / d_{\mathrm{S}}$ ratios than the earlystep genes, contrary to the pattern found in anthocyanin pathway genes [13]. Particularly, the ENC data indicated that silent sites were more constrained (lower ENC values) in the downstream than in the upstream genes (table 1 ), which would overestimate $d_{N} / d_{S}$ ratios of the downstream genes. This observation suggests that the difference of selective constraints between the downstream and upstream genes would be much more striking.

As indicated by Cork and Purugganan [17], the specific nature of selection on the component genes depended largely on the function of the pathway but the dichotomy between upstream and downstream genes in a pathway was a crude differentiation of function. In our case, no correlation between evolutionary rate and position of genes in the GA pathway seems better explained by the pathway fluxes hypothesis, suggesting that natural selection more likely target enzymes that control metabolic fluxes $[17,18]$. As predicted by this theory, the enzymes carrying high metabolic fluxes (e.g., at branch points of the pathway) will control disproportionately the expression of biochemical phenotypes and experience higher evolutionary constraints $[8,14,17]$. In the GA pathway, enzymes that catalyze multiple steps or at the branching points (KO, KAO, GA20ox2, GA3ox, and GA2ox) have also low $d_{N} / d_{S}$ values (Figures 1 and 2 ). In contrast, the gene with the highest $d_{\mathrm{N}} / d_{\mathrm{S}}$ encodes the enzyme involving in a single step (KS) in the pathway. It should be noted that upstream enzymes in many pathways are positioned above major branch points and thus suffer from stronger selection constraints due to pleiotropic effects $[5,17]$. This may partly explain, in terms of pathway fluxes theory, the differences in levels of selective constraints upon upstream and downstream genes observed in earlier stud- 
ies $[9,11,13,15]$. In addition, we found that the genes at the branching points (e.g., GA20ox2 and GA3ox2) have high levels of gene expression (usually measured by codon usage bias) (table 1) and slow $d_{\mathrm{N}}$ (Figure 2). Such a correlation in the GA pathway also supports the functional hypothesis that more highly expressed protein will consume a larger proportion of the cell resources and might control more important metabolic fluxes [6]. This explanation is in well agreement with studies on the GA pathway genes in other plants. For example, increased expression of a GA20ox gene in transgenic Arabidopsis plants caused an increase in GA levels and GA overdose phenotypes while overexpression of the CPS in Arabidopsis (AtCPS) did not affect the levels of bioactive GAs or the phenotype $[24,40]$. It should be noted that translation selection and selection for protein folding might be also a factor contributing to differences of evolutionary rates $[7,21]$. However, our case cannot provide additional evidence because of limited number of genes used and lack of information on the protein structure and expression of these genes.

\section{Rate variation among GA genes is attributed to selective constraint rather than positive selection}

As discussed above, we excluded the possibility that difference of mutation rates was the main factor for rate variation of the GA pathway genes. However, high $d_{\mathrm{N}} / d_{\mathrm{S}}$ ratio for some genes (e.g., KS1, KO2) might be due to relaxation of selection and could also be explained by repeated adaptive selection on these proteins. Earlier studies have showed that evolutionary rate variation among proteins might be attributed either to the differences in the magnitude of selective constraints $[1,11,15,41]$ or to the differences in the frequency of positive selection [37]. In spite of many investigations on factors influencing rate of protein evolution $[5,6,8,14,17]$, few have been conducted to explore the relative contribution of differential selective constraint and differential positive selection to rate variation of pathway genes $[13,15]$. In this study, both branch and codon models failed to detect any signature of positive selection for any lineage and codon of the GA pathway genes, suggesting that increased rate of nonsynonymous substitution in some genes is mainly ascribed to relaxed selective constraint, in agreement with the studies on anthocyanin pathway genes $[13,15]$. Unlike some empirical studies [14], however, we did not detect adaptive substitution for those genes that encode enzymes controlling metabolic fluxes.

Studies showed that highly expressed genes were more important to an organism and thus subject to greater selective constraint and these genes usually had greater codon bias because of selection for translation efficiency $[5,13,33,43,44]$. A recent study on a set of six distantly related model organisms confirmed that translational selection would be an important mechanism behind the constraints of proteins by increasing translational accuracy and translational robustness [21]. Therefore, the degree to which a gene is subject to selective constraint could also be reflected by the magnitude of codon usage bias in that gene. Indeed, GA200x2 that is identical to the rice Green Revolution gene, Semi-Dwarf1 [23], has the lowest nonsynonymous substitution rate (the smallest $d_{\mathrm{N}} / d_{\mathrm{S}}$ ratio) among 7 genes surveyed. This gene exhibits the strongest codon usage bias (the lowest ENC), consistent with its functional importance. In contrast, the gene (KS1) that encodes the enzyme involving a single step in the pathway has the highest $d_{\mathrm{N}} / d_{\mathrm{S}}$ ratio and much weaker codon usage bias (much higher ENC). Consequently, the significant heterogeneity of evolutionary rate of the GA pathway genes is mainly ascribed to differential constraint relaxation rather than the positive selection. It should be noted, however, that the power to detect positive selection using the above methods may be low, particularly when adaptive substitutions are distributed across many amino acid sites such that $d_{\mathrm{N}} / d_{\mathrm{S}}$ is not elevated above 1 for any site $[15,45]$. Further investigations with alternative tests on within-species variation $[10,12,14,15]$ would be necessary to detect evidence of positive selection and elucidate the differential rate of adaptive substitution among the GA pathway genes.

\section{Conclusion}

Based on sequence analyses of 7 core structural genes of the gibberellin biosynthetic pathway, we have detected significant rate heterogeneity among these genes for both synonymous and nonsynonymous sites and ascribed such rate variation to differences of selection intensity rather than differential mutation pressures on the genes. Our study demonstrates that the downstream genes in the GA pathway did not exhibit the elevated substitution rate, inconsistent with previous argument that downstream genes in metabolic pathways would evolve more rapidly than upstream genes. Instead, we found that the GA pathway genes encoding either the enzyme at the branch point or enzymes catalyzing multiple steps had the lowest evolutionary rates because of strong purifying selection, supporting the pathway flux theory that predicts that natural selection primarily targets enzymes that have the greatest control on fluxes.

\section{Abbreviations}

GA: gibberellin; ENC: effective number of codons; LRT: likelihood ratio test.

\section{Authors' contributions}

SG and YHY designed the research and outlined the manuscript together. YHY and SG performed the research. YHY and FMZ analyzed the data. SG, YHY and ZFM inter- 
preted the data and wrote the paper. All authors have read and approved the final manuscript.

\section{Additional material}

\section{Additional file 1}

figure S1. Phylogeny of the rice tribe (Oryzeae) obtained from the com bined Adh 2 and GPA1 sequences by Bayesian inference under $\operatorname{Tr} \mathrm{N}+\mathrm{G}$ model [26]. Bold faces indicate the species sampled in this study. Click here for file

[http://www.biomedcentral.com/content/supplementary/14712148-9-206-S1.doc]

\section{Additional file 2}

figure S2. Schematic diagrams of 7 GA biosynthetic genes and the regions sequenced in this study. Boxes and lines indicate exons and introns, respectively. Exon numbers are labeled with the roman numbers. Locations of primers for each fragment are showed above the diagrams and their sequences are provided in table 1.

Click here for file

[http://www.biomedcentral.com/content/supplementary/14712148-9-206-S2.doc]

\section{Additional file 3}

table S1. Species used in this study.

Click here for file

[http://www.biomedcentral.com/content/supplementary/14712148-9-206-S3.doc]

\section{Additional file 4}

table S2. Information of the 7 GA genes sampled in the present study. Click here for file

[http://www.biomedcentral.com/content/supplementary/14712148-9-206-S4.doc]

\section{Additional file 5}

table S3. Sequences and melting temperatures (Tm) of internal primers used for sequencing of four genes that are $\sim 2 \mathrm{~kb}$ in length.

Click here for file

[http://www.biomedcentral.com/content/supplementary/1471-

2148-9-206-S5.doc]

\section{Additional file 6}

figure S3. Pruned phylogeny tree consisting of 6 species. Branches $b 1$ to b10 are 10 independent branches.

Click here for file

[http://www.biomedcentral.com/content/supplementary/14712148-9-206-S6.doc]

\section{Additional file 7}

table S4. Wilcoxon signed rank test of pairwise comparisons of $\mathrm{d}_{N}$ and $\mathrm{d}_{S}$ between genes for 10 independent branches of the pruned tree consisting of 6 species.

Click here for file

[http://www.biomedcentral.com/content/supplementary/14712148-9-206-S7.doc]

\section{Additional file 8}

table S5. Log-likelihood values, $\omega$ ratios and parameter estimates under models of variable $\omega$ ratios among codon sites.

Click here for file

[http://www.biomedcentral.com/content/supplementary/14712148-9-206-S8.doc]

\section{Acknowledgements}

We thank Xin-Hui Zou, Hong-Zheng Sun and other members of Ge's group for their helps during the experiment and data analyses, and Yingqing Lu for helpful suggestions. We acknowledge the International Rice Research Institute (Los Banos, Philippines) for providing seed samples. This work was supported by the National Basic Research Program of China (2007CB815704), National Natural Science Foundation of China (30I2 1003, 30430030), and the grants from the Chinese Academy of Sciences.

\section{References}

I. Li WH: Molecular evolution. Sinauer Associates, Sunderland, Mass; 1997.

2. Tiffin $P$, Hahn MW: Coding sequence divergence between two closely related plant species: Arabidopsis thaliana and Brassica rapa ssp. pekinensis. J Mol Evol 2002, 54(6):746-753.

3. Mclnerney JO: The causes of protein evolutionary rate variation. Trends Ecol Evol 2006, 2 I (5):230-232.

4. Plotkin JB, Fraser HB: Assessing the determinants of evolutionary rates in the presence of noise. Mol Biol Evol 2007, 24(5): $1113-1|2|$.

5. Pal C, Papp B, Lercher MJ: An integrated view of protein evolution. Nat Rev Genet 2006, 7(5):337-348.

6. Rocha EP: The quest for the universals of protein evolution. Trends Genet 2006, 22(8):4I2-4I6.

7. Drummond DA, Raval A, Wilke CO: A single determinant dominates the rate of yeast protein evolution. Mol Biol Evol 2006, 23(2):327-337.

8. Vitkup D, Kharchenko P, Wagner A: Influence of metabolic network structure and function on enzyme evolution. Genome Biol 2006, 7(5):R39.

9. Riley RM, Jin W, Gibson G: Contrasting selection pressures on components of the Ras-mediated signal transduction pathway in Drosophila. Mol Ecol 2003, I 2(5): | |3 | $5-1323$.

10. Whitt SR, Wilson LM, Tenaillon MI, Gaut BS, Buckler ESt: Genetic diversity and selection in the maize starch pathway. Proc Natl Acad Sci USA 2002, 99(20): 12959-1 2962.

II. Rausher MD, Miller RE, Tiffin P: Patterns of evolutionary rate variation among genes of the anthocyanin biosynthetic pathway. Mol Biol Evol 1999, I6(2):266-274.

12. Olsen KM, Womack A, Garrett AR, Suddith JI, Purugganan MD: Contrasting evolutionary forces in the Arabidopsis thaliana floral developmental pathway. Genetics 2002, 160(4): I64|-1650.

13. Lu $Y$, Rausher MD: Evolutionary rate variation in anthocyanin pathway genes. Mol Biol Evol 2003, 20(II): | 844-I853.

14. Flowers JM, Sezgin E, Kumagai S, Duvernell DD, Matzkin LM, Schmidt PS, Eanes WF: Adaptive evolution of metabolic pathways in Drosophila. Mol Biol Evol 2007, 24(6): I347-I354.

15. Rausher M, Lu Y, Meyer K: Variation in constraint versus positive selection as an explanation for evolutionary rate variation among anthocyanin genes. J MolEvol 2008, 67(2): I37-144.

16. Ramsay H, Rieseberg LH, Ritland K: The correlation of evolutionary rate with pathway position in plant terpenoid biosynthesis. Mol Biol Evol 2009, 26(5): 1045-1053.

17. Cork JM, Purugganan MD: The evolution of molecular genetic pathways and networks. Bioessays 2004, 26(5):479-484

18. Eanes WF: Analysis of selection on enzyme polymorphisms. Annu Rev Ecol Syst 1999, 30(I):301-326.

19. Li H, Helling R, Tang C, Wingreen N: Emergence of preferred structures in a simple model of protein folding. Science 1996, 273(5275):666-669. 
20. Bloom JD, Drummond DA, Arnold FH, Wilke CO: Structural determinants of the rate of protein evolution in yeast. Mol Biol Evol 2006, 23(9): |75|-|76I.

2I. Drummond DA, Wilke CO: Mistranslation-induced protein misfolding as a dominant constraint on coding-sequence evolution. Cell 2008, I34(2):34I-352.

22. Hahn M, Conant $G$, Wagner A: Molecular evolution in large genetic networks: Does connectivity equal constraint? J Mol Evol 2004, 58(2):203-2II.

23. Sakamoto T, Miura K, Itoh H, Tatsumi T, Ueguchi-Tanaka M, Ishiyama K, Kobayashi M, Agrawal GK, Takeda S, Abe K, et al.: An overview of gibberellin metabolism enzyme genes and their related mutants in rice. Plant Physiol 2004, I 34(4): I642-I653.

24. Yamaguchi S: Gibberellin metabolism and its regulation. Annu Rev Plant Biol 2008, 59(I):225-25I.

25. Ge S, Sang T, Lu BR, Hong DY: Phylogeny of rice genomes with emphasis on origins of allotetraploid species. Proc Natl Acad Sci USA 1999, 96(25): |4400-| 4405.

26. Guo YL, Ge S: Molecular phylogeny of Oryzeae (Poaceae) based on DNA sequences from chloroplast, mitochondrial, and nuclear genomes. Am J Bot 2005, 92(9): I 548-I 558.

27. GPWG: Phylogeny and subfamilial classification of the grasses (Poaceae). Ann Missouri Bot Gard 200I, 88(3):373-457.

28. Thompson JD, Gibson TJ, Plewniak F, Jeanmougin F, Higgins DG: The CLUSTAL_X windows interface: flexible strategies for multiple sequence alignment aided by quality analysis tools. Nucl Acids Res 1997, 25(24):4876-4882.

29. Xia X, Xie Z: DAMBE: software package for data analysis in molecular biology and evolution. J Hered 200I, 92(4):37I-373.

30. Yang Z, Nielsen R: Estimating synonymous and nonsynonymous substitution rates under realistic evolutionary models. Mol Biol Evol 2000, I 7(I):32-43.

31. Yang Z: PAML 4: phylogenetic analysis by maximum likelihood. Mol Biol Evol 2007, 24(8): I586-|59|.

32. Sharp PM, Tuohy TM, Mosurski KR: Codon usage in yeast: cluster analysis clearly differentiates highly and lowly expressed genes. Nucl Acids Res 1986, I 4( I3):5 I 25-5 I 43.

33. Sharp PM: Determinants of DNA sequence divergence between Escherichia coli and Salmonella typhimurium: codon usage, map position, and concerted evolution. J Mol Evol I99I, 33(I):23-33

34. Rozas J, Rozas R: DnaSP version 3: an integrated program for molecular population genetics and molecular evolution analysis. Bioinformatics 1999, I 5(2): I74- I75.

35. Wright F: The "effective number of codons" used in a gene. Gene 1990, 87(I):23-29.

36. Wilcoxin F: Individual comparisons by ranking methods. Biometrics 1945, I(6):80-83.

37. Yang Z, Bielawski JP: Statistical methods for detecting molecular adaptation. Trends Ecol Evol 2000, I 5(I 2):496-503

38. Yang Z, Nielsen R: Synonymous and nonsynonymous rate variation in nuclear genes of mammals. J Mol Evol 1998 46(4):409-4I8.

39. Bielawski JP, Yang Z: Maximum likelihood methods for detecting adaptive evolution after gene duplication. I Struct funct Genom 2003, 3(I-4):20I-2I2

40. Yang Z: Computational molecular evolution. Oxford: Oxford University Press; 2006.

4I. Gillespie JH: The causes of molecular evolution. Oxford: Oxford University Press; 1991.

42. Wolfe $\mathrm{KH}$, Sharp $\mathrm{PM}, \mathrm{Li}$ WH: Mutation rates differ among regions of the mammalian genome. Nature 1989, 337(6204):283-285.

43. Powell JR, Moriyama EN: Evolution of codon usage bias in Drosophila. Proc Natl Acad Sci USA 1997, 94( I 5):7784-7790.

44. Popescu CE, Borza T, Bielawski JP, Lee RW: Evolutionary rates and expression level in Chlamydomonas. Genetics 2006 , I 72(3): I567-I576.

45. Kosakovsky Pond SL, Frost SDW: Not so different after all: a comparison of methods for detecting amino acid sites under selection. Mol Biol Evol 2005, 22(5): I 208-I 222.
Publish with Bio Med Central and every scientist can read your work free of charge

"BioMed Central will be the most significant development for disseminating the results of biomedical research in our lifetime. "

Sir Paul Nurse, Cancer Research UK

Your research papers will be:

- available free of charge to the entire biomedical community

- peer reviewed and published immediately upon acceptance

- cited in PubMed and archived on PubMed Central

- yours - you keep the copyright
BioMedcentral 